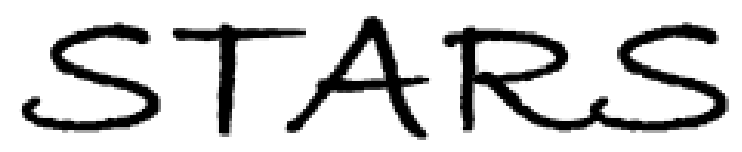

University of Central Florida

STARS

$1-1-1989$

\title{
Modulational Instability Of Electrostatic Drift Waves In An Inhomogeneous-Plasma
}

Bhimsen K. Shivamoggi

University of Central Florida

Find similar works at: https://stars.library.ucf.edu/facultybib1980

University of Central Florida Libraries http://library.ucf.edu

This Note is brought to you for free and open access by the Faculty Bibliography at STARS. It has been accepted for inclusion in Faculty Bibliography 1980 s by an authorized administrator of STARS. For more information, please contact STARS@ucf.edu.

\section{Recommended Citation}

Shivamoggi, Bhimsen K., "Modulational Instability Of Electrostatic Drift Waves In An InhomogeneousPlasma" (1989). Faculty Bibliography 1980s. 841.

https://stars.library.ucf.edu/facultybib1980/841

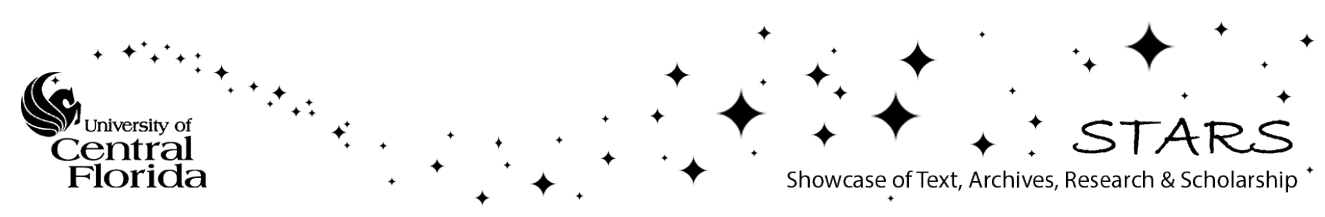




\title{
Modulational instability of electrostatic drift waves in an inhomogeneous plasma
}

\author{
Bhimsen K. Shivamoggi \\ University of Central Florida, Orlando, Florida 32816 \\ (Received 12 December 1988; revised manuscript received 23 February 1989)
}

\begin{abstract}
In this Brief Report, we investigate the modulational instability of electrostatic drift waves in an inhomogeneous plasma with respect to quasi-one-dimensional modulations that primarily propagate along the drift direction (like the drift solitons given by Lakhin, Mikhailovskii, and Onishchenko [Phys. Lett. A 119, 348 (1987); Plasma Phys. Contr. Fusion 30, 457 (1988)]).
\end{abstract}

Nonlinear drift waves in a low- $\beta$, nonuniform plasma embedded in a magnetic field with frequency $\omega$ much below the ion-cyclotron frequency $\omega_{c i}$ and wave vector $\mathbf{k}$ in the direction almost perpendicular to the magnetic field have been studied in connection with enhanced diffusion in magnetic confinement devices (Hasegawa and co-workers, ${ }^{1,2}$ Nozaki and co-workers $\left.{ }^{3,4}\right)$. Experiments (Mazzucato, ${ }^{5}$ and Slusher and Surko ${ }^{6}$ ) indicated the existence of quasi-three-dimensional drift waves. Hasegawa and Mima $^{1}$ gave a simple model equation to describe electrostatic drift turbulence of the above type in a twodimensional $\mathbf{E} \times \mathbf{B}$ guiding center plasma. Lakhin et al..$^{7,8}$ showed that when only the quadratic nonlinearity is kept, the Hasegawa-Mima equation possesses driftsoliton solutions. The drift solitons have been observed in a $Q$ machine (Buchelnikova et al., ${ }^{9}$ Hendel et al., ${ }^{10}$ and Politzer ${ }^{11}$ ). [The $Q$ machine has a magnetically confined plasma consisting of ions produced by surface ionization of $\mathrm{Cs}$ or $\mathrm{K}$ atomic beams incident on hot tungsten ionizer (end) plates at both ends of the plasma column and of thermionic electrons emitted from these plates.] In this paper we will investigate the modulational instability of drift waves in an inhomogeneous plasma with respect to more realistic general quasi-onedimensional perturbations that primarily propagate along the drift direction (like the drift solitons ${ }^{7,8}$ ); the previous treatments (Nozaki et al. ${ }^{3}$ and Majumdar ${ }^{12}$ ) of this aspect considered modulations that are two dimensional with the simplifying assumption of periodicity in the density-gradient direction.

Consider an electron-ion plasma in an external magnetic field $B_{0} \hat{i}_{z}$. The ions are taken to be cold, to be stratified in the $x$ direction, i.e., $n_{0}=n_{0}(x)$, and to move two dimensionally in a plane perpendicular to the magnetic field. The electrons are taken to be hot and to move three dimensionally. We then have the ion-continuity equation together with the Boltzmann distribution for the electrons (which remain in equilibrium while responding to the low-frequency turbulence), and the quasineutrality condition. The electrostatic potential $\phi$ then satisfies the following equation (Hasegawa et al., ${ }^{2}$ Nozaki et al., ${ }^{3}$ and Majumdar ${ }^{12}$ ):

$$
\begin{aligned}
\frac{\partial}{\partial t}\left(\nabla_{\perp}^{2} \phi-\phi\right)-\left(\nabla_{\perp} \phi \times \hat{\mathbf{i}}_{z}\right) \cdot \nabla\left(\nabla_{\perp}^{2} \phi-\ln n_{0}\right) & \\
& +\nabla \cdot\left(\phi \nabla \phi_{t}\right)=0,
\end{aligned}
$$

where the subscript $\perp$ refers to the component perpendicular to the magnetic field, and the various quantities have been nondimensionalized as follows (in usual notations):

$$
x^{\prime}=x / \rho_{i}, \quad t^{\prime}=\omega_{c i} t, \quad \phi^{\prime}=e \phi / k T_{e}
$$

and the primes have been dropped subsequently. It should be noted that in Eq. (1) the scalar nonlinear term is actually of higher order than the vector nonlinear term and should be treated as such in the perturbation treatment. However, as in Majumdar, ${ }^{12}$ this is not necessary because, for the plane waves considered in the present formulation, it turns out that to $O\left(\epsilon^{2}\right), \epsilon$ being the typical wave amplitude, the vector nonlinear term makes a zero contribution, while the scalar nonlinear term makes the whole contribution.

Let us consider the density distribution of the form

$$
n_{0}(x) \sim e^{\sigma x}
$$

so that Eq. (1) becomes

$$
\begin{array}{r}
\frac{\partial}{\partial t}\left(\frac{\partial^{2} \phi}{\partial x^{2}}+\frac{\partial^{2} \phi}{\partial y^{2}}-\phi\right)-\left(\frac{\partial \phi}{\partial y} \frac{\partial}{\partial x}-\frac{\partial \phi}{\partial x} \frac{\partial}{\partial y}\right)\left(\frac{\partial^{2} \phi}{\partial x^{2}}+\frac{\partial^{2} \phi}{\partial y^{2}}\right) \\
+\sigma \frac{\partial \phi}{\partial y}+\phi\left(\frac{\partial^{3} \phi}{\partial x^{2} \partial t}+\frac{\partial^{3} \phi}{\partial y^{2} \partial t}\right) \\
+\left(\frac{\partial \phi}{\partial x} \frac{\partial^{2} \phi}{\partial x \partial t}+\frac{\partial \phi}{\partial y} \frac{\partial^{2} \phi}{\partial y \partial t}\right)=0
\end{array}
$$

Let us look for quasi-one-dimensional modulations that primarily propagate in the $y$ direction (which is the drift direction), and so introduce the following independent variables:

$$
\xi=k y-\omega t, \quad \xi=\epsilon(y-c t), \quad \eta=\epsilon x, \quad \tau=\epsilon^{2} t, \quad \epsilon \ll 1
$$

where $c=d \omega / d k$ is the group velocity of the primary traveling wave.

Equation (3) then becomes 


$$
\begin{aligned}
-\omega( & \left.k^{2} \phi_{\xi \xi \xi}-\phi_{\xi}\right)+k \sigma \phi_{\xi}-k^{2} \omega\left(\phi \phi_{\xi \xi \xi}+\phi_{\xi} \phi_{\xi \xi}\right) \\
+ & \epsilon\left[-c\left(k^{2} \phi_{\xi \xi \xi}-\phi_{\xi}\right)+\sigma \phi_{\xi}-2 k \omega \phi_{\xi \xi \xi}+k^{3}\left(\phi_{\eta} \phi_{\xi \xi \xi}-\phi_{\xi} \phi_{\xi \xi \eta}\right)-k \omega\left(2 \phi \phi_{\xi \xi \xi}+\phi_{\xi} \phi_{\xi \xi}+\phi_{\xi} \phi_{\xi \xi}\right)-k^{2} c\left(\phi \phi_{\xi \xi \xi}+\phi_{\xi} \phi_{\xi \xi}\right)\right] \\
+ & \epsilon^{2}\left[-\omega \phi_{\eta \eta \xi}+k^{2} \phi_{\xi \xi \tau}-\phi_{\tau}-2 k c \phi_{\xi \xi \xi}-k^{2}\left(\phi_{\zeta} \phi_{\xi \xi \eta}+2 \phi_{\xi} \phi_{\xi \xi \eta}+3 \phi_{\eta} \phi_{\xi \xi \zeta}\right)\right. \\
& \left.\quad-\omega\left(\phi \phi_{\eta \eta \xi}+\phi_{\eta} \phi_{\eta \xi}+\phi \phi_{\zeta \xi \xi}+\phi_{\zeta} \phi_{\xi \xi}\right)-k c\left(2 \phi \phi_{\xi \zeta \xi}+\phi_{\xi} \phi_{\zeta \xi}+\phi_{\zeta} \phi_{\xi \xi}\right)+k^{2}\left(\phi \phi_{\xi \xi \tau}+\phi_{\xi} \phi_{\xi \tau}\right)\right]=0 .
\end{aligned}
$$

Let us now look for a solution of the form

$$
\phi(x, y, t)=\sum_{n=1}^{\infty} \epsilon^{n} \phi_{n}(\xi, \zeta, \eta, \tau) .
$$

Equation (5) then gives a hierarchy of problems of various orders for $\epsilon-0(\epsilon)$,

$$
-\omega\left(k^{2} \phi_{1_{\xi \xi \xi}}-\phi_{1_{\xi}}\right)+k \sigma \phi_{1_{\xi}}=0
$$

for $O\left(\epsilon^{2}\right)$,

$$
-\omega\left(k^{2} \phi_{2_{\xi \xi \xi}}-\phi_{2_{\xi}}\right)+k \sigma \phi_{2_{\xi}}=c\left(k^{2} \phi_{1_{\xi \xi \xi}}-\phi_{1_{\xi}}\right)-\sigma \phi_{1_{\xi}}-2 k \omega \phi_{1_{\xi \xi \xi}}+k^{2} \omega\left(\phi_{1} \phi_{1_{\xi \xi \xi}}+\phi_{1_{\xi}} \phi_{1_{\xi \xi}}\right) ;
$$

and for $O\left(\epsilon^{3}\right)$,

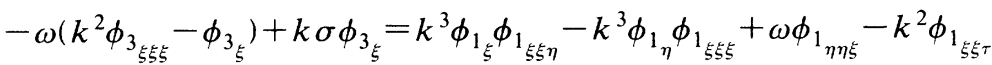

$$
\begin{aligned}
& +\phi_{1_{\tau}}+2 k c \phi_{1_{\xi \xi \xi}}+k^{2} \omega\left(\phi_{1} \phi_{2_{\xi \xi \xi}}+\phi_{2} \phi_{1_{\xi \xi \xi}}+\phi_{1_{\xi}} \phi_{2_{\xi \xi}}+\phi_{2_{\xi}} \phi_{1_{\xi \xi}}\right) \\
& +k \omega\left(2 \phi_{1} \phi_{1_{\xi \xi \xi}}+\phi_{1_{\xi}} \phi_{1_{\xi \xi}}+\phi_{1_{\xi}} \phi_{1_{\xi \xi}}\right)+k^{2} c\left(\phi_{1} \phi_{1_{\xi \xi \xi}}+\phi_{1_{\xi}} \phi_{1_{\xi \xi}}\right) \text {. }
\end{aligned}
$$

We obtain from Eq. (7) the linear solution

$$
\begin{aligned}
& \phi_{1}=A_{1}(\zeta, \eta, \tau) e^{i \xi}+\text { c.c. }, \\
& \omega=-k \sigma /\left(k^{2}+1\right) .
\end{aligned}
$$

Using (10), Eq. (8) becomes

$$
\begin{aligned}
-\omega\left(k^{2} \phi_{2_{\xi \xi \xi}}-\phi_{2_{\xi}}\right)+k \sigma \phi_{2_{\xi}} \\
=-\frac{3 k^{2} \sigma}{\left(k^{2}+1\right)} A_{1_{\xi}} e^{i \xi}-2 i k^{2} \omega A_{1}^{2} e^{2 i \xi}+\text { c. c. }
\end{aligned}
$$

Removal of the secular terms in Eq. (11) requires

$$
A_{1_{\xi}}=0 \text {, }
$$

or

$$
A_{1}=A_{1}(\eta, \tau) \text {. }
$$

We then have

$$
\phi_{2}=-\frac{1}{3} A_{1}^{2} e^{2 i \xi}+\text { c.c. }
$$

Using (10), (12), and (13), Eq. (9) becomes

$$
\begin{gathered}
-\omega\left(k^{2} \phi_{3_{\xi \xi \xi}}-\phi_{3_{\xi}}\right)+k \sigma \phi_{3_{\xi}} \\
=-\frac{i k \sigma}{k^{2}+1} A_{1_{\eta \eta}} e^{i \xi}+\left(k^{2}+1\right) A_{1_{\tau}} e^{i \xi} \\
-\frac{5 i k^{3} \sigma}{3\left(k^{2}+1\right)}\left|A_{1}\right|^{2} A_{1} e^{i \xi}+\text { c.c. }
\end{gathered}
$$

Removal of the scalar terms in Eq. (14) leads to the nonlinear Schrödinger equation

$i A_{1_{\tau}}+\frac{k \sigma}{\left(k^{2}+1\right)^{2}} A_{1_{\eta \eta}}+\frac{5 k^{3} \sigma}{3\left(k^{2}+1\right)^{2}}\left|A_{1}\right|^{2} A_{1}=0$.

Note that the product of the coefficients of the dispersion and nonlinear terms is

$$
\frac{k \sigma}{\left(k^{2}+1\right)^{2}} \frac{5 k^{3} \sigma}{3\left(k^{2}+1\right)^{2}}=\frac{5 k^{4} \sigma^{2}}{3\left(k^{2}+1\right)^{4}}>0 .
$$

Therefore we conclude that the drift waves are unstable with respect to quasi-one-dimensional modulations of the form (6) for all values of the wave number $k$. This is in contrast to the situation for the two-dimensional modulations considered by Nozaki et al. ${ }^{3}$ and Majumdar, ${ }^{12}$ which are unstable only for $k$ lying in a certain window in the wave-number space. Consequently, the present modulations are more important in the nonlinear development of the drift waves. The onset of this modulation instability then leads to localized structures (the envelope solitons) perpendicular to the applied magnetic field, which are believed to produce an enhanced particle diffusion in the plasma. 
${ }^{1}$ A. Hasegawa and K. Mima, Phys. Fluids 21, 87 (1978).

${ }^{2}$ A. Hasegawa, C. A. MaClennan, and Y. Kodama, Phys. Fluids 22, 2122 (1979).

${ }^{3}$ K. Nozaki, T. Taniuti, and K. Watanabe, J. Phys. Soc. Jpn. 46, 983 (1979).

${ }^{4}$ K. Nozaki, Phys. Rev. Lett. 46, 184 (1981).

${ }^{5}$ E. Mazzucato, Phys. Rev. Lett. 36, 792 (1976).

${ }^{6}$ R. E. Slusher and C. M. Surko, Phys. Rev. Lett. 37, 1747 (1976).

${ }^{7}$ V. P. Lakhin, A. B. Mikhailovskii, and O. G. Onishchenko,
Phys. Lett. A 119, 348 (1987).

${ }^{8}$ V. P. Lakhin, A. B. Mikhailovskii, and O. G. Onishchenko, Plasma Phys. Contr. Fusion 30, 457 (1988).

${ }^{9}$ N. S. Buchelnikova, R. A. Salimov, and J. I. Edelman, Zh. Eksp. Teor. Fiz. 52, 837 (1967) [Sov. Phys._JETP 25, 548 (1967)].

${ }^{10}$ H. W. Hendel, T. K. Chu, and P. A. Politzer, Phys. Fluids 11, 2426 (1968).

${ }^{11}$ P. A. Politzer, Phys. Fluids 14, 2156 (1971).

${ }^{12}$ D. Majumdar, J. Plasma Phys. 40, 253 (1988). 